\title{
Dissipative dynamics of matter-wave solitons in a nonlinear optical lattice
}

\author{
F. Kh. Abdullaev, ${ }^{1}$ A. Gammal, ${ }^{2}$ H. L. F. da Luz, ${ }^{2}$ and Lauro Tomio ${ }^{1, *}$ \\ ${ }^{1}$ Instituto de Física Teórica, Universidade Estadual Paulista, 01405-900, São Paulo, Brazil \\ ${ }^{2}$ Instituto de Física, Universidade de São Paulo, 05315-970, São Paulo, Brazil
}

(Received 1 July 2007; published 11 October 2007)

\begin{abstract}
Dynamics and stability of solitons in two-dimensional (2D) Bose-Einstein condensates (BEC), with onedimensional (1D) conservative plus dissipative nonlinear optical lattices, are investigated. In the case of focusing media (with attractive atomic systems), the collapse of the wave packet is arrested by the dissipative periodic nonlinearity. The adiabatic variation of the background scattering length leads to metastable matterwave solitons. When the atom feeding mechanism is used, a dissipative soliton can exist in focusing 2D media with 1D periodic nonlinearity. In the defocusing media (repulsive BEC case) with harmonic trap in one direction and nonlinear optical lattice in the other direction, the stable soliton can exist. Variational approach simulations are confirmed by full numerical results for the 2D Gross-Pitaevskii equation.
\end{abstract}

DOI: 10.1103/PhysRevA.76.043611

PACS number(s): 03.75.Lm, 05.30.Jp

\section{INTRODUCTION}

The dynamics of optical and matter-wave solitons with different type of management of system parameters has been under intensive investigations in the past years $[1,2]$. Two types of modulations have been considered: Dispersion and nonlinearity management, which can both occur in time and space. Temporal strong and rapid modulations of the dispersion are more interesting in optical fibers due to many advantages of dispersion managed solitons for optical communications and for storage of information [3-5]. Temporal modulations of the nonlinearity are promising in fiber ring lasers and Bose-Einstein condensates (BEC) [6-8]. In the latter case, several recent works have been done discussing the suppression of collapse, leading to the existence of stable multidimensional solitons in attractive BEC, and with the generation of periodic patterns of matter waves [9-17]. In optics, nonlinearity managed solitons have also been observed, as described in Refs. [18-21].

In view of the actual experimental possibilities, recent attention has also been devoted to periodic spatial management in nonlinear optics and Bose-Einstein condensates. In optical media, the nonlinear Kerr coefficient can be periodically modulated in space, leading to the problem of an optical beam in a two-dimensional (2D) medium with nonlinearity management. In BEC, the spatial variation of scattering length is possible [22-28], for example, by using optically induced Feshbach resonance [29,30]. In the 2D case, the situation is less clear. The study of a one-dimensional (1D) nonlinear periodic potential in a 2D nonlinear Schrödinger equation (NLSE) shows that broad solitons are unstable. As verified in Ref. [31], narrow solitons centered on the maximum of the lattice potential can be stable, but the stability region is so narrow that they are physically unstable. Stable gap solitons can exist in BEC under combination of linear and nonlinear periodic potentials [32-34]. However, in general the models considered till now are strongly idealized. In particular, using the optically induced Feshbach resonances

\footnotetext{
*Corresponding author: tomio@ift.unesp.br
}

we can generate a mixture of conservative and dissipative nonlinear optical lattices. In view of that, around the Feshbach resonance one can observe nonvanishing contributions of the imaginary part of the scattering length.

In the present work, after an analysis of a conservative system with nonlinear optical lattice, we consider the influence of nonlinear dissipation on the dynamics and the stability of solitons. In particular, we note that the role of such kind of dissipation can be crucial for the existence of solitons in multidimensional nonlinear optical lattices. Such hope is supported by the well-known fact that homogeneous nonlinear dissipations can arrest collapse in the cubic focusing multidimensional NLSE [35]. The possibility of existence of dissipative solitons is investigated considering compression effects and atom feeding. For the stability analysis of the solutions we consider the Vakhitov-Kolokolov (VK) criterion [36], which has been discussed in Refs. [37,38], complemented by numerical simulations.

The organization of the paper is as follows. The model is described in the next section. In Sec. III, we investigate the properties of localized states in the cases of attractive and repulsive 2D condensates in 1D nonlinear optical lattice, with or without harmonic trap in one of the dimensions. In Sec. IV, we perform an analysis of the evolution of a 2D soliton under 1D periodic nonlinearity and dissipation, using the variational approach and by direct numerical simulation of the GP equation.

\section{MODEL}

Recently, the generation of nonlinear optical lattices in BEC by two counterpropagating laser beams near the optical induced Feshbach resonance has been suggested $[25,26]$. The spatial variation of the optical intensity leads to a spatial periodic variation of the atomic scattering length. Such structure can support new types of localized nonlinear states. Considering the minimum requirements of having spatial periodic variation in the nonlinear cubic term of a $2 \mathrm{D}$ GP equation, for the wave function $\psi \equiv \psi\left(x_{1}, x_{2}, t\right)$ we can have the following expression: 


$$
i \hbar \frac{\partial \psi}{\partial t}=-\frac{\hbar^{2}}{2 m}\left(\frac{\partial^{2} \psi}{\partial x_{1}^{2}}+\beta \frac{\partial^{2} \psi}{\partial x_{2}^{2}}\right)-g\left(x_{1}, x_{2}\right)|\psi|^{2} \psi
$$

with

$$
g\left(x_{1}, x_{2}\right) \equiv \frac{g_{0}}{2}+\left(g_{1}+i g_{2}\right)\left[\cos ^{2}\left(k x_{1}\right)+\delta_{0} \cos ^{2}\left(k x_{2}\right)\right] .
$$

In the above equations $\beta=0$ (1) for the 1D (2D) case with $\delta_{0}$ parametrizing the anisotropy of the nonlinear optical lattice in the $2 \mathrm{D}$ space $\left(\delta_{0}=0\right.$, if the optical lattice is given only in the $x_{1}$ direction, and $=1$ when the lattice geometry is the same in both directions). $g_{0}$ is related to the $s$-wave two-body scattering length $a_{s}, g_{0} \equiv\left(4 \pi \hbar^{2} / m\right) a_{s}$, with $g_{0}>0 \quad\left(g_{0}<0\right)$ for attractive (repulsive) condensates; $g_{1}(>0)$ is related to the optical intensity; and $g_{2}$ parametrizes dissipative effects.

The optically induced scattering length and the inelastic collision rate coefficient $K_{\text {inel }}$ (imaginary part of $a_{s}$ ) are described by [29]

$$
\begin{gathered}
\operatorname{Re}\left(a_{s}\right)=a_{s 0}+\frac{1}{2 k_{i}}\left[\frac{\Gamma_{\text {stim }}(x) \Delta}{\Delta^{2}+\left(\Gamma_{\text {spon }} / 2\right)^{2}}\right], \\
K_{\text {inel }} \equiv \operatorname{Im}\left(a_{s}\right)=\frac{2 \pi \hbar}{m} \frac{1}{k_{i}}\left[\frac{\Gamma_{\text {stim }}(x) \Gamma_{\text {spon }}}{\Delta^{2}+\left(\Gamma_{\text {spon }} / 2\right)^{2}}\right],
\end{gathered}
$$

where $a_{s 0}$ is the background two-body scattering length, $\Delta$ is the detuning from the photoassociated resonance, and $\hbar k_{i}$ is the relative momentum of the collision. $\Gamma_{\text {stim }}$ is the resonant transition rate between the continuum and the molecular state [proportional to the laser intensity $I(x)$ ], with $\Gamma_{\text {spon }}$ being the spontaneous decay rate from the excited molecular state. Far from the resonance, the imaginary part of the scattering length is small, such that $\operatorname{Im}(a) \ll \operatorname{Re}(a)$. In Ref. [30], it was shown that in the experiment with ${ }^{87} \mathrm{Rb}$ one can obtain optically induced large variations of the scattering length. The laser intensity was $460 \mathrm{~W} / \mathrm{cm}^{2}$ and the variations $a_{s}$ occurred from $10 a_{0}$ to $190 a_{0}$ (with $a_{s 0}=100 a_{0}, a_{0}$ being the Bohr radius).

By considering the following variable changes and definitions in Eq. (1),

$$
\begin{gathered}
\kappa x=2 k x_{1}, \quad \kappa y=2 k x_{2}, \quad \tau=\frac{4 w_{R} t}{\kappa^{2}}, \\
w_{R}=\frac{E_{R}}{\hbar}, \quad E_{R}=\frac{\hbar^{2} k^{2}}{2 m}, \quad \gamma_{i=0,1,2}=\frac{g_{i}}{2\left|g_{0}\right|},
\end{gathered}
$$

we obtain the dimensionless equation

$$
i \frac{\partial u}{\partial \tau}+\frac{\partial^{2} u}{\partial x^{2}}+\beta \frac{\partial^{2} u}{\partial y^{2}}+\gamma(x, y)|u|^{2} u=0,
$$

where

$$
\gamma(x, y) \equiv \gamma_{0}+\left(\gamma_{1}+i \gamma_{2}\right)\left[1+\delta_{0}+\cos (\kappa x)+\delta_{0} \cos (\kappa y)\right],
$$

and the wave function was redefined such that

$$
u \equiv u(x, y, \tau)=\sqrt{\frac{\kappa^{2}\left|g_{0}\right|}{4 E_{R}}} \psi
$$

From Eq. (6) to Eq. (9), we should note that $\gamma_{0}$ is fixed to $-1 / 2$ for attractive condensates; and $+1 / 2$ for repulsive condensates. Different cases can be considered: Full 1D geometry is realized when $\beta=\delta_{0}=0$. The anisotropic $2 \mathrm{D}$ case is realized for $\beta=1$ and $\delta_{0}=0$. And the $2 \mathrm{D}$ isotropic case can be achieved with $\beta=\delta_{0}=1$. Next, we consider more explicitly in our study the anisotropic 2D case, with $\beta=1$ and $\delta_{0}=0$. As the soliton is completely free in the $y$ direction, we also examine the possibility of having a harmonic trap $m \omega_{2}^{2} x_{2}^{2} / 2$. Following the transformations (5), a dimensionless frequency $\omega$ is also defined, such that

$$
\omega \equiv \kappa^{2} \frac{\omega_{2}}{8 w_{R}}, \quad \frac{m}{2} \omega_{2}^{2} x_{2}^{2}=\left(\frac{4 E_{R}}{\kappa^{2}}\right) \omega^{2} y^{2} .
$$

In order to extend our study of the stability conditions to a few realistic cases, the effect of a compression is also verified, which can be achieved by a time variation of the background value of the scattering length [22], leading to a timedependent $\gamma_{0}$. To achieve this purpose, we use the following time-dependent expression for $\gamma_{0}$ :

$$
\gamma_{0} \rightarrow \gamma_{0}(\tau)=\gamma_{0} \exp \left[2 \alpha\left(\tau-\tau_{c}\right) \theta\left(\tau-\tau_{c}\right)\right],
$$

where $\theta$ is the Heaviside function $[\theta(x)=0(1)$ for $x<0 \quad(x$ $>0)$ ] controlling the initial time when the compression is switched on. Compression effect, achieved by a feeding process, can be described by an additional linear term $i \alpha_{f} u$ in the GP equation [39]. If the modulation of nonlinearity in time is induced by increasing the transverse frequency of the trap, then we should multiply the full nonlinear term by $\exp (2 \alpha \tau)$. With these considerations, Eq. (7) can be written as

$$
i \frac{\partial u}{\partial \tau}=-\frac{\partial^{2} u}{\partial x^{2}}-\frac{\partial^{2} u}{\partial y^{2}}-\gamma(x, \tau)|u|^{2} u+\omega^{2} y^{2} u+i \alpha_{f} u,
$$

where

$$
\gamma(x, \tau) \equiv \gamma_{0}(\tau)+\left(\gamma_{1}+i \gamma_{2}\right)[1+\cos (\kappa x)] .
$$

In the above, one should take $\alpha_{f}=0$ when $\alpha \neq 0$ in Eq. (11), as such parameters have a similar role in the formalism.

\section{CONSERVATIVE SYSTEM}

It is useful to describe shortly the solitons and their stability in the conservative case $\left(\gamma_{2}=0\right)$. The one-dimensional conservative case has been considered by using a variational approach (VA) in [26]. Using an exact approach, the 2D case with 1D nonlinear optical lattice was studied in [31], where the authors have considered the case with attractive background nonlinearity $\left(\gamma_{1}>0\right)$. Looking for perspective applications to BEC, we will consider here the 2D problem with a 1D nonlinear optical lattice. Following Ref. [26], we start our analysis using the VA formalism.

With $u(x, y, \tau) \equiv v(x, y) \exp (-i \mu \tau)$ in Eq. (12), and taking $\alpha_{f}, \alpha$, and $\gamma_{2}$ equal to zero, we obtain 


$$
\mu v=-\frac{\partial^{2} v}{\partial x^{2}}-\frac{\partial^{2} v}{\partial y^{2}}-\left[\widetilde{\gamma}_{0}+\gamma_{1} \cos (\kappa x)\right] v^{3}+\omega^{2} y^{2} v,
$$

where we are redefining $\gamma_{0}$ to $\tilde{\gamma}_{0} \equiv \gamma_{0}+\gamma_{1}$. In view of our definitions in Eq. (5), this implies that for attractive condensed systems we have $\widetilde{\gamma}_{0}=\gamma_{1}+1 / 2$ and, for repulsive ones, $\tilde{\gamma}_{0}=\gamma_{1}-1 / 2$. The sign of $\tilde{\gamma}_{0}$ gives the sign of the background field. But, we should note that we can have situations where the same $\tilde{\gamma}_{0} \geq 0$ can refer to attractive or repulsive condensates. For example: $\tilde{\gamma}_{0}=1$ with $\gamma_{1}=1 / 2$ and $\gamma_{0}=1 / 2$ (attractive); $\widetilde{\gamma}_{0}=1$ with $\gamma_{1}=3 / 2$ and $\gamma_{0}=-1 / 2$ (repulsive).

These two situations differ in Eq. (14), because the strength of the oscillatory term is different. However, as the results are similar, we prefer to analyze separately the cases of repulsive condensates with negative background field, which occur when $0<\gamma_{1}<1 / 2\left(0>\widetilde{\gamma}_{0} \geq-1 / 2\right)$.

The corresponding averaged Lagrangian $L$ is obtained from the density $\mathcal{L}$ as

$$
\begin{gathered}
L=\int_{-\infty}^{\infty} d x \int_{-\infty}^{\infty} d y \mathcal{L} \\
2 \mathcal{L}=\mu v^{2}-\left|\frac{\partial v}{\partial x}\right|^{2}-\left|\frac{\partial v}{\partial y}\right|^{2}+\frac{\tilde{\gamma}_{0}+\gamma_{1} \cos (\kappa x)}{2} v^{4}-\omega^{2} y^{2} v^{2} .
\end{gathered}
$$

Here, it is interesting to observe that a scaling given by $\kappa$ is applied to the observables obtained from the above equations as the root-mean-square radius in $x$ and $y$ directions, chemical potentials, frequencies, and energies. In order to see that, we can redefine all the observables using the variable transformation, $\bar{x} \equiv \kappa x$ and $\bar{y} \equiv \kappa y$, such that we have no $\kappa$ dependence in a new set of observables (represented with a "bar") that are being calculated. This scaling essentially implies considering $\kappa \equiv 1$ in all the equations. At the end, the physical observables will be given by the relations (5) (with $\kappa$ $=1$ ). For example, in the case of mean-square radius we will have

$$
\left\langle x_{1}^{2}\right\rangle=\frac{\left\langle x^{2}\right\rangle}{4 k^{2}}, \quad\left\langle x_{2}^{2}\right\rangle=\frac{\left\langle y^{2}\right\rangle}{4 k^{2}} .
$$

Next, in our VA we consider the Gaussian ansatz

$$
v(x, y)=A \exp \left(-\frac{x^{2}}{2 a_{1}^{2}}-\frac{y^{2}}{2 a_{2}^{2}}\right),
$$

where $A$ is the amplitude and $a_{i}(i=1,2)$ the corresponding widths in the $x$ and $y$ directions, respectively. $N=\pi a_{1} a_{2} A^{2}$ is the normalization of Eq. (18), with the corresponding averaged Lagrangian given by

$$
\begin{aligned}
L= & \int_{-\infty}^{\infty} d x \int_{-\infty}^{\infty} d y \mathcal{L}=\frac{N}{2}\left[\mu-\left(\frac{1}{2 a_{1}^{2}}+\frac{1}{2 a_{2}^{2}}\right)-\frac{\omega^{2} a_{2}^{2}}{2}\right. \\
& \left.+\frac{N}{4 \pi a_{1} a_{2}}\left(\widetilde{\gamma}_{0}+\gamma_{1} e^{-\kappa^{2} a_{1}^{2} / 8}\right)\right] .
\end{aligned}
$$

From the Euler-Lagrange equations for the parameters, $\partial L / \partial N=0$ and $\partial L / \partial a_{i=1,2}=0$, we obtain

$$
\begin{gathered}
2 \mu=\frac{1}{a_{1}^{2}}+\frac{1}{a_{2}^{2}}-\frac{N}{\pi a_{1} a_{2}}\left(\widetilde{\gamma}_{0}+\gamma_{1} e^{-\kappa^{2} a_{1}^{2} / 8}\right)+\omega^{2} a_{2}^{2}, \\
N=\frac{4 \pi a_{2}}{a_{1}\left[\tilde{\gamma}_{0}+\gamma_{1} e^{-\kappa^{2} a_{1}^{2} / 8}\left(1+\frac{\kappa^{2} a_{1}^{2}}{4}\right)\right]}, \\
\omega^{2} a_{2}^{4}+\frac{a_{2}^{2}}{a_{1}^{2}} \frac{\left(\tilde{\gamma}_{0}+\gamma_{1} e^{-\kappa^{2} a_{1}^{2} / 8}\right)}{\left[\tilde{\gamma}_{0}+\gamma_{1} e^{-\kappa^{2} a_{1}^{2} / 8}\left(1+\frac{\kappa^{2} a_{1}^{2}}{4}\right)\right]}-1=0 .
\end{gathered}
$$

In the case that $\omega=0$, this set of equations, for $\mu, N$, and $a_{2}$, can be expressed in terms of $a_{1}$, as

$$
\begin{gathered}
\mu_{0}=-\frac{1}{a_{1}^{2}}\left(\frac{\tilde{\gamma}_{0}+\gamma_{1} e^{-\kappa^{2} a_{1}^{2} / 8}\left[1-\frac{\kappa^{2} a_{1}^{2}}{8}\right]}{\tilde{\gamma}_{0}+\gamma_{1} e^{-\kappa^{2} a_{1}^{2} / 8}\left[1+\frac{\kappa^{2} a_{1}^{2}}{4}\right]}\right), \\
a_{2,0} \equiv a_{1} \sqrt{\frac{\tilde{\gamma}_{0}+\gamma_{1} e^{-\kappa^{2} a_{1}^{2} / 8\left(1+\frac{\kappa^{2} a_{1}^{2}}{4}\right)}}{\tilde{\gamma}_{0}+\gamma_{1} e^{-\kappa^{2} a_{1}^{2} / 8}}}, \\
N_{0}=\frac{4 \pi}{\sqrt{\left[\tilde{\gamma}_{0}+\gamma_{1} e^{-\kappa^{2} a_{1}^{2} / 8}\left(1+\frac{\kappa^{2} a_{1}^{2}}{4}\right)\right]\left[\tilde{\gamma}_{0}+\gamma_{1} e^{-\kappa^{2} a_{1}^{2} / 8}\right]}} .
\end{gathered}
$$

For the case that $\omega \neq 0$, the relation for $a_{2}$ in terms of $a_{1}$ can be obtained from Eqs. (22) and (24):

$$
a_{2}=\frac{1}{\omega a_{2,0}} \sqrt{\left[\sqrt{\frac{1}{4}+\omega^{2} a_{2,0}^{4}}-\frac{1}{2}\right]} .
$$

Equations (26), (20), and (21) form the set of equations for $\omega \neq 0$.

Next, we consider separately the cases of attractive systems, with $\tilde{\gamma}_{0}=\gamma_{1}+\frac{1}{2}>0$, and repulsive ones, with $\tilde{\gamma}_{0}=\gamma_{1}$ $-\frac{1}{2}<0$.

\section{A. Attractive condensate $\left(\widetilde{\gamma}_{0}=\gamma_{1}+1 / 2\right)$}

This case, which corresponds to $\gamma_{0}=1 / 2$ and $\gamma_{1}>0$, has been investigated recently in [31]. With $\omega=0$, it is applied to the set of Eqs. (23), (25), and (24). In the general case with $\omega \neq 0$, we should consider Eqs. (20), (21), and (26).

Let us first verify the analytic limiting cases of the VA expressions for $\omega=0$,

$$
\begin{gathered}
a_{2,0} \rightarrow a_{1} \quad \text { for } a_{1} \ll 1 \text { and } a_{1} \gg 1, \\
\mu_{0} \rightarrow-1 / a_{1}^{2} \text { for } a_{1} \ll 1 \text { and } a_{1} \gg 1, \\
N_{0} \rightarrow \frac{4 \pi}{\tilde{\gamma}_{0}+\gamma_{1}}=\frac{4 \pi}{2 \gamma_{1}+1 / 2} \text { for } a_{1}=0, \\
N_{0} \rightarrow \frac{4 \pi}{\tilde{\gamma}_{0}}=\frac{4 \pi}{\gamma_{1}+1 / 2} \text { for } a_{1} \rightarrow \infty,
\end{gathered}
$$

and the limiting cases of the VA expressions for $\omega \neq 0$,

$$
a_{2} \rightarrow\left\{\begin{array}{l}
a_{1} \text { for } a_{1} \ll 1, \\
1 / \sqrt{\omega} \text { for } a_{1} \gg 1,
\end{array}\right.
$$



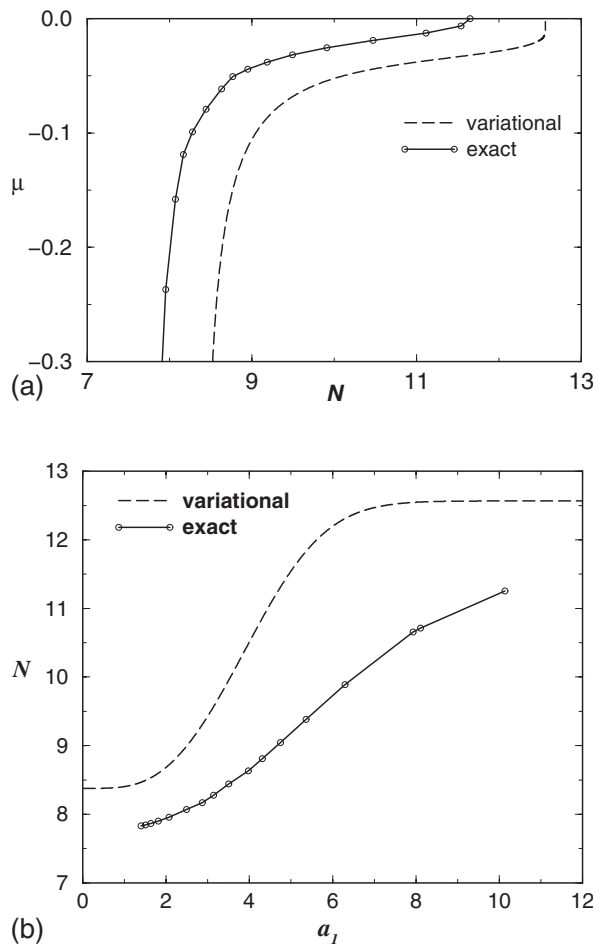

FIG. 1. Attractive case, with $\tilde{\gamma}_{0}=1$ and $\gamma_{1}=0.5$. Results for the chemical potential $\mu$, as a function of $N$ (upper frame) and $N$ vs $a_{1}$ (lower frame), obtained using variational approach (VA) and full numerical calculations. The variational parameter for the width, $a_{1}$, and the root-mean-square radius, $\sqrt{\left\langle x^{2}\right\rangle}$, are related by $a_{1}=\sqrt{2\left\langle x^{2}\right\rangle}$ [Actually, the physical observables depend on $k$ as given by Eqs. (5) and (17)].

$$
\begin{gathered}
\mu \rightarrow\left\{\begin{array}{l}
-1 / a_{1}^{2} \text { for } a_{1} \ll 1, \\
\omega \quad \text { for } a_{1} \gg 1,
\end{array}\right. \\
N \rightarrow \frac{4 \pi}{\tilde{\gamma}_{0}+\gamma_{1}} \text { for } a_{1}=0, \\
N \rightarrow \frac{4 \pi a_{2}}{\tilde{\gamma}_{0} a_{1}} \rightarrow 0 \text { for } a_{1} \rightarrow \infty .
\end{gathered}
$$

In Fig. 1, we plot the corresponding results for the chemical potential $\mu$ as a function of $N$ (upper frame) and $N$ as a function of $a_{1}$ (lower frame). Numerical solutions to PDE results were done with the algorithm presented in scheme $\mathrm{C}$ of Ref. [40]. Considering the Vakhitov-Kolokolov (VK) criterion [36] expressing the necessary condition for the soliton stability (given by $d \mu / d N<0$ ), we observe that the results given in the upper frame of Fig. 1 provide evidence that the solitons are not stable. The soliton is orbitally stable if the orbit near the ground state remains close to this state for all times. In view of some limitations on the applicability of this criterion, as discussed in Sec. V of Ref. [38], the actual conclusions on the stability were also verified by numerical simulations. These results are in agreement with the prediction of Ref. [31].
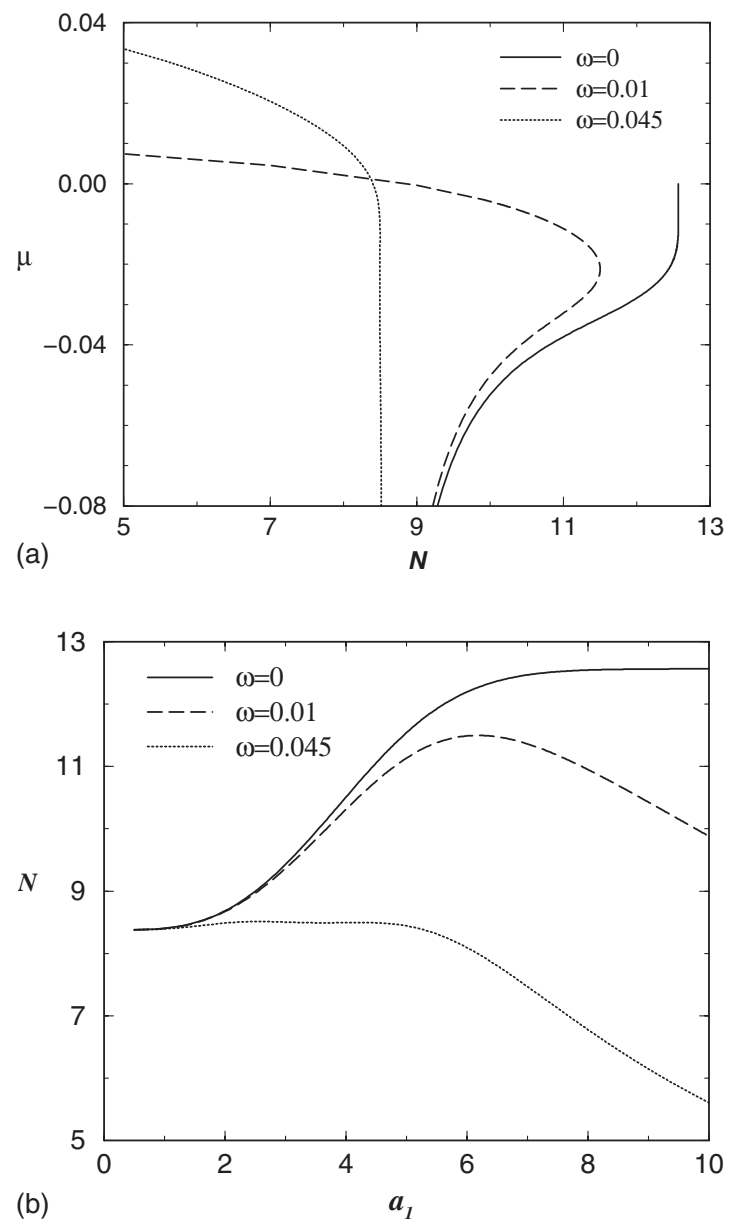

FIG. 2. Attractive case, with $\widetilde{\gamma}_{0}=1$ and $\gamma_{1}=0.5$. VA results for the chemical potential $\mu$, as a function of $N$ (upper frames) and $N$ vs $a_{1}$ (lower frame). The results are given for $\mu$ near zero, considering three values of the frequency: $\omega=0$ (solid line), 0.01 (dashed line), and 0.045 (dotted line).

We note, from the VA results, that in the limit of large $a_{1}$ the system has a tendency to stabilize, indicating that with just a small trapping potential we can produce a stable region. This behavior is shown by the VA results given in Fig. 2 . The variational approach, besides an expected small quantitative shift, provides a good qualitative picture of the results when compared with full numerical predictions. If one is first concerned with the stability of the system (instead of the quantitative results of the observables), the VA provides a nice and reliable picture.

In our VA, when we keep $\omega$ fixed (zero or nonzero) and increase the value of $\gamma_{1}$, we observe that the general picture in respect to stability of the system does not change. This leads us to conclude that we cannot improve the stability of the system by increasing the strength of the lattice periodicity for attractive condensates. In the following, we are going to analyze the cases with $\tilde{\gamma}_{0}<0$.

\section{B. Repulsive condensate with $\tilde{\gamma}_{0}<0\left(\tilde{\gamma}_{0}=\gamma_{1}-1 / 2\right)$}

We should recall that by repulsive condensate we mean an atomic system where the particles have originally positive 
two-body scattering length, such that in Eq. (2) we have $g_{0}$ $<0$ or $\gamma_{0}=-1 / 2$. So, given $\gamma_{1}$ (parameter of the spatial periodic variation of the atomic scattering length), $\tilde{\gamma}_{0}=\gamma_{1}$ $-1 / 2$. And, if we also consider a negative background, such that $\widetilde{\gamma}_{0}<0, \gamma_{1}$ will be restricted to the interval $0<\gamma_{1}<1 / 2$.

Some other limitations are applied in the parameters, considering that the widths and $N$ must be real positive quantities. The relation between the widths $a_{2}$ and $a_{1}$, Eq. (24) for $\omega=0$, implies a limitation to the values of $a_{1}$ :

$$
e^{-\kappa^{2} a_{1}^{2} / 8} \geq \frac{1}{2 \gamma_{1}}-1, \quad \rightarrow a_{1, \max }^{2}=\frac{8}{\kappa^{2}} \ln \left(\frac{\gamma_{1}}{\frac{1}{2}-\gamma_{1}}\right) .
$$

This limit, $a_{1, \max }$, is necessary in order to have $a_{2}$ and $N$ real and positive quantities for any value of $\omega$. It will also restrict the actual values of the parameter $\gamma_{1}$ to $1 / 4<\gamma_{1}<1 / 2$. The cases with $\gamma_{1}>1 / 2$ are also allowed, in principle, without upper limit for $a_{1}$. However, such cases will correspond to a positive background field, $\widetilde{\gamma}_{0}>0$, that has already been considered in the preceding subsection. In view of the above, let us also verify for this case the analytic VA limits.

For $\omega=0$

$$
\begin{gathered}
a_{2,0} \rightarrow \begin{cases}a_{1} \text { for } a_{1} \ll 1, \\
\infty & \text { for } a_{1}=a_{1, \max },\end{cases} \\
\mu_{0} \rightarrow \begin{cases}-1 / a_{1}^{2} & \text { for } a_{1} \ll 1, \\
1 /\left(2 a_{1}^{2}\right) & \text { for } a_{1}=a_{1, \max },\end{cases} \\
N_{0} \rightarrow \begin{cases}8 \pi /\left(4 \gamma_{1}-1\right) & \text { for } a_{1}=0, \\
\infty & \text { for } a_{1}=a_{1, \max }\end{cases}
\end{gathered}
$$

and for $\omega \neq 0$

$$
\begin{aligned}
& a_{2} \rightarrow \begin{cases}a_{1} & \text { for } a_{1} \ll 1, \\
1 / \sqrt{\omega} & \text { for } a_{1}=a_{1, \max },\end{cases} \\
& \mu \rightarrow \begin{cases}-1 / a_{1}^{2} & \text { for } a_{1} \ll 1, \\
1 /\left(2 a_{1}^{2}\right)+\omega & \text { for } a_{1}=a_{1, \max },\end{cases} \\
& N \rightarrow \begin{cases}8 \pi /\left(4 \gamma_{1}-1\right) & \text { for } a_{1}=0, \\
32 \pi /\left[\left(1-2 \gamma_{1}\right) \sqrt{\omega} \kappa^{2} a_{1, \max }^{3}\right] & \text { for } a_{1}=a_{1, \max } .\end{cases}
\end{aligned}
$$

In Fig. 3, we plot $N$ vs $a_{1}$ and the chemical potential $\mu$ vs $N$, for $\widetilde{\gamma}_{0}=-0.1$ and $\gamma_{1}=0.4$, considering VA and four values of $\omega(0,0.07,0.1,0.3)$. In the case of $\omega=0$, we also include results obtained from exact PDE calculations. Following the VK criterion for stability, $d \mu / d N<0$, we notice that stable regions start to appear with $\omega \approx 0.1$. With $\omega>0.3 \kappa^{2}$ the unstable regions almost disappear. However, as one can observe in the lower frame, the width $a_{1}$ is quite limited due to the condition (27). The observables $\mu$ and $a_{i}$ depend on the wave parameter $k$ of to the spatial periodic variation of the atomic scattering length through the scaling relations (5) and (6) with $\kappa=1$. However, contrary to some discussions and conclusions of Ref. [31], specific values of the parameter $k$ cannot affect the conclusions on stability. In such cases of conservative systems, the stability results from combined effects given by the parameters $\tilde{\gamma}_{0}, \gamma_{1}$, and $\omega$. Our main con-
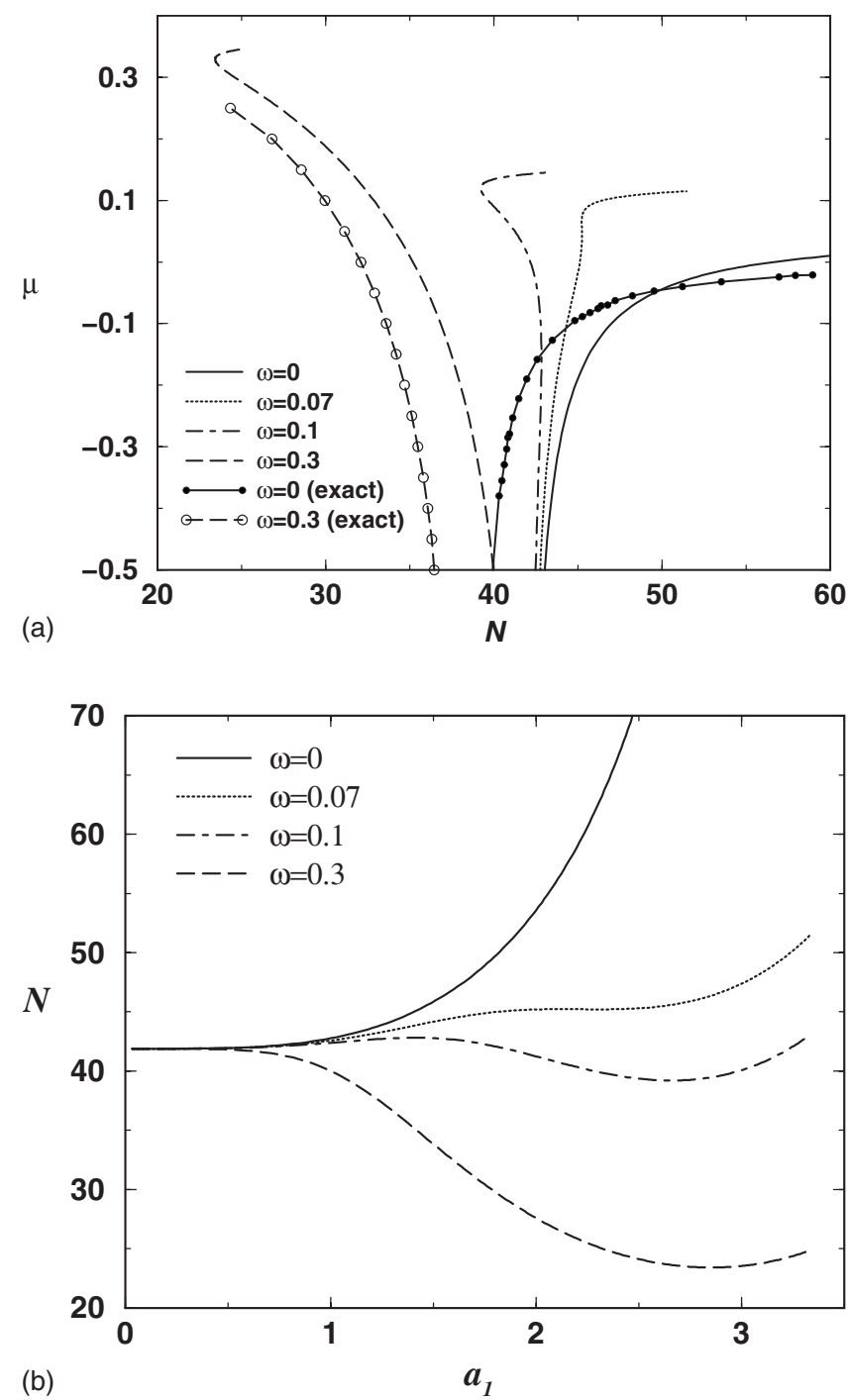

FIG. 3. Repulsive case, with $\widetilde{\gamma}_{0}=-0.1$ and $\gamma_{1}=0.4$, for $\mu$ vs $N$ (upper frame), and $N$ vs $a_{1}$ (lower frame). In both frames, we show the results using the variational approach, for $\omega=0,0.07,0.1$, and 0.3 . In the upper frame, the exact PDE results are also shown in two cases: $\omega=0$ (for which the system is unstable) and $\omega=0.3$ (for which the system is stable). In this last case, near the region where the VA presents a small unstable branch $(22<N<25)$, our exact numerical results are shown only for $N>24$. As observed, the VA is giving a general picture of the exact solutions.

clusion is that, without the trapping potential (included in the $y$ direction), taking $\omega=0$, the optical lattice cannot stabilize the solutions, neither for repulsive nor for attractive condensates.

In order to further check the role of the optical lattice, for the repulsive case we also investigate the case with constant $\omega$ and different values of $\gamma_{1}$. From the results shown in Fig. 3 , for $\gamma_{1}=0.4$, we found appropriate to consider $\omega=0.07$, which has a marginal stability near $\mu \approx 0.05$. The results are shown in Fig. 4, where we first observe that a larger $\gamma_{1}$ can help to allow the width $a_{1}$ to increase, within the limiting condition (27). However, the marginal stability remains for corresponding different values of the chemical potential. In 

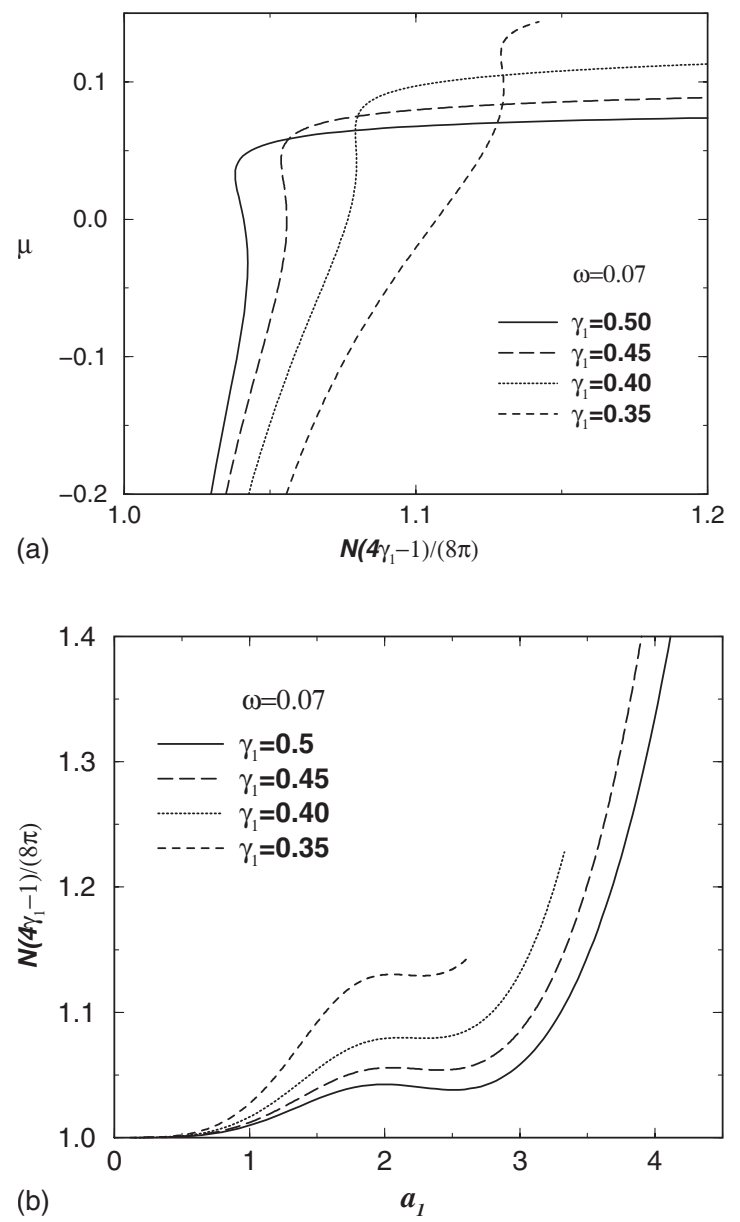

FIG. 4. VA results for the repulsive case, with $\tilde{\gamma}_{0}=-0.1$ and $\omega$ fixed to 0.07 , considering $\gamma_{1}=0.35,0.4,0.45$, and 0.5 . In the upper frame we have $\mu$ vs $N / N\left(a_{1}=0\right)$ and, in the lower frame, $N / N\left(a_{1}\right.$ $=0)$ vs $a_{1}$.

order to keep the plots of Fig. 4 for different values of $\gamma_{1}$ in the same frames, we have normalized the number $N$ such that it is equal to one when $a_{1}$ is zero.

The plots of the profiles are presented in Fig. 5 for $\omega=0$ and 0.3 . Stability is verified by the numerical evolution confirming the VK prediction. The profiles at $t=50$ are practically undistinguishable from the initial form, in good agreement with the VA.

\section{EVOLUTION OF 2D SOLITON UNDER 1D PERIODIC NONLINEARITY AND DISSIPATION}

In this section, we will consider the case $\gamma_{2} \neq 0$ we have in Eq. (12). To study the dynamics of a 2D soliton with 1D periodic nonlinearity and dissipation, we also apply a variational approach and full numerical calculations. In the Gaussian ansatz (18), we should also include parameters related to dissipative effects and initial conditions. As dissipative solitons are known to be chirped for a bright soliton [41], the ansatz can be taken in the following form:

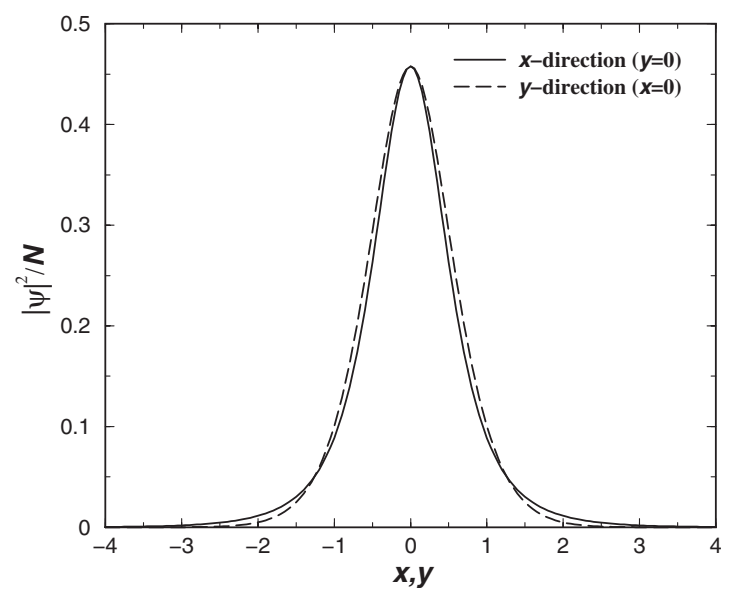

FIG. 5. The soliton profile in the stable region predicted by the VK criterion for $\omega=0.3, \widetilde{\gamma}_{0}=-0.1, \gamma_{1}=0.4, \mu=0.1$, and $N \approx 33$ at the time $t=50$. Solid line is $x$ direction; dashed line is $y$ direction.

$$
\begin{aligned}
u= & A \exp \left[-\frac{\left(x-x_{0}\right)^{2}}{2 a_{1}^{2}}-\frac{y^{2}}{2 a_{2}^{2}}\right] \exp \left[i b_{1}\left(x-x_{0}\right)^{2}+i b_{2} y^{2}\right. \\
& +i \phi(\tau)] .
\end{aligned}
$$

In this case, we include the chirp parameters $b_{i}(i=1,2)$, a coordinate parameter in $x$ direction given by $x_{0}$, and a phase $\phi$ of the wave packet.

The Lagrangian density for Eq. (12) is

$$
\mathcal{L}(x, \tau)=\frac{i}{2}\left(u_{\tau} u^{*}-u_{\tau}^{*} u\right)-\left|u_{x}\right|^{2}-\left|u_{y}\right|^{2}+\frac{\gamma(x)}{2}|u|^{4},
$$

where $\gamma(x)$ is given by Eq. (13). Next, from the ansatz (28), we obtain the corresponding averaged Lagrangian:

$$
\begin{aligned}
L= & \int_{-\infty}^{\infty} d x \int_{-\infty}^{\infty} d y \mathcal{L}=-\frac{\pi}{2} A^{2} a_{1} a_{2}\left[a_{1}^{2}\left(b_{1 \tau}+4 b_{1}^{2}\right)+\frac{1}{a_{1}^{2}}\right. \\
& +a_{2}^{2}\left(b_{2 \tau}+4 b_{2}^{2}\right)+\frac{1}{a_{2}^{2}}+2 \phi_{\tau}-\frac{A^{2}}{2}\left[\widetilde{\gamma}_{0}\right. \\
& \left.\left.+\gamma_{1} \cos \left(\kappa x_{0}\right) e^{-\kappa^{2} a_{1}^{2} / 8}\right]\right] .
\end{aligned}
$$

The equations for the soliton parameters $\eta_{i}=[A, a, b, \phi]$ in the VA are derived from (see for example [42])

$$
\frac{\partial L}{\partial \eta_{i}}-\frac{d}{d \tau} \frac{\partial L}{\partial \eta_{i, \tau}}=\int_{-\infty}^{\infty} d x \int_{-\infty}^{\infty} d y\left[R \frac{\partial u^{*}}{\partial \eta_{i}}+\text { c.c. }\right],
$$

where the perturbation term $R$ is

$$
R=-i \gamma_{2}[1+\cos (\kappa x)]|u|^{2} u+i \alpha_{f} u .
$$

Here we are taking into account a linear amplification term $\left(\alpha_{f}\right)$ describing the atoms feeding. Finally, from the above, we obtain the following system of five coupled ordinary differential equations (ODE) to be solved for the parameters of our variational approach (VA): 


$$
\begin{array}{rl}
\left(A^{2} a_{1} a_{2}\right)_{\tau}= & -\gamma_{2} A^{4} a_{1} a_{2} e^{-\kappa^{2} a^{2} / 8} \cos \left(\kappa x_{0}\right)-\gamma_{2} A^{4} a_{1} a_{2} \\
& +2 \alpha_{f} A^{2} a_{1} a_{2}, \\
\left(A^{2} a_{1}^{3} a_{2}\right)_{\tau}= & 8 A^{2} a_{1}^{3} a_{2} b_{1}-\frac{\gamma_{2}}{2} A^{4} a_{1}^{3} a_{2}\left[1+\frac{1}{4} \cos \left(\kappa x_{0}\right)(4\right. \\
& \left.\left.-\kappa^{2} a_{1}^{2}\right) e^{-\kappa^{2} a_{1}^{2} / 8}\right]+2 \alpha_{f} A^{2} a_{1}^{3} a_{2}, \\
\left(A^{2} a_{1} a_{2}^{3}\right)_{\tau}= & 8 A^{2} a_{1} a_{2}^{3} b_{2}-\frac{\gamma_{2}}{2} A^{4} a_{2}^{3} a_{1}\left[1+e^{-\kappa^{2} a_{1}^{2} / 8} \cos \left(\kappa x_{0}\right)\right] \\
& +2 \alpha_{f} A^{2} a_{1} a_{2}^{3}, \\
b_{1 \tau}=\frac{1}{a_{1}^{4}}-4 & 4 b_{1}^{2}-\frac{\tilde{\gamma}_{0} A^{2}}{4 a_{1}^{2}}-\frac{\gamma_{1} A^{2}}{4 a_{1}^{2}} \cos \left(\kappa x_{0}\right) e^{-\kappa^{2} a_{1}^{2} / 8}\left(1+\frac{\kappa^{2} a_{1}^{2}}{4}\right), \\
b_{2 \tau}= & \frac{1}{a_{2}^{4}}-4 b_{2}^{2}-\frac{\tilde{\gamma}_{0} A^{2}}{4 a_{2}^{2}}-\frac{\gamma_{1} A^{2}}{4 a_{2}^{2}} \cos \left(\kappa x_{0}\right) e^{-\kappa^{2} a_{1}^{2} / 8} .
\end{array}
$$

By taking into account that the norm $N=\pi A^{2} a_{1} a_{2}$, with $i, j$ $=1,2(i \neq j)$, we have

$$
\begin{gathered}
N_{\tau}=-\frac{\gamma_{2} N^{2}}{\pi a_{1} a_{2}}\left[1+e^{-\kappa^{2} a_{1}^{2} / 8} \cos \left(\kappa x_{0}\right)\right]+2 \alpha_{f} N, \\
\left(a_{i}^{2}\right)_{\tau}=8 a_{i}^{2} b_{i}+\frac{\gamma_{2} N a_{i}}{2 \pi a_{j}}\left[1+e^{-\kappa^{2} a_{1}^{2} / 8} \cos \left(\kappa x_{0}\right)\left(1+\delta_{i, 1} \frac{\kappa^{2} a_{1}^{2}}{4}\right)\right], \\
b_{i, \tau}=\frac{1}{a_{i}^{4}}-4 b_{i}^{2}-\frac{N}{4 \pi a_{i}^{3} a_{j}}\left\{\gamma_{0}+\gamma_{1}\left[1+e^{-\kappa^{2} a_{1}^{2} / 8} \cos \left(\kappa x_{0}\right)\right.\right. \\
\left.\left.\times\left(1+\delta_{i, 1} \frac{\kappa^{2} a_{1}^{2}}{4}\right)\right]\right\} .
\end{gathered}
$$

Next, we present some of our results, when considering periodic nonlinearity with dissipative effects. Considering the scaling of observables with $\kappa$, discussed for the conservative systems in Sec. III, which can also be verified in the present case, we have the corresponding transformation $b_{i} \rightarrow b_{i} / \kappa^{2}$.

In Fig. 6, we have results for the full numerical simulations (PDE) for the evolution of the matter-wave packet under combination of the conservative and dissipative nonlinear optical lattice in the case of the attractive condensate $\gamma_{0}=1 / 2$. As we can see the collapse is arrested by the dissipative nonlinear optical lattice. The results are compared with the prediction of the VA approach (ODE). We observe a good agreement of the VA with full numerical calculations.

We also have investigated the role of a deviation $\delta$ of the given norm from the critical norm, in the initial wave packet: $A \rightarrow A(1+\delta)$. The results of the full numerical simulations are presented in Fig. 7. Increasing the deviation $\delta$ from the critical norm, multiple peaks are observed, corresponding to revivals of the wave packet during the collapse. The number of peaks grows as $\delta$ varies from 0.02 to 0.5 . The focusing-

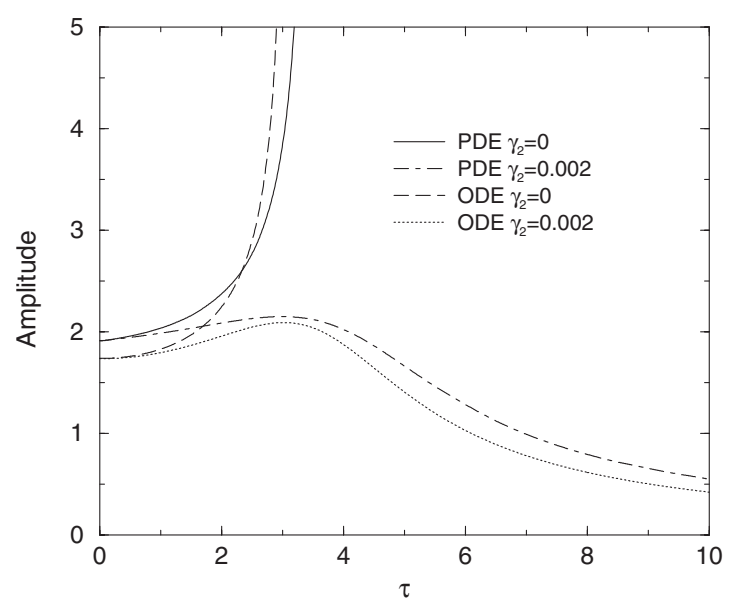

FIG. 6. Results for the amplitude (A) as a function of $\tau$, using variational approach (VA) and full numerical calculations, for the attractive case with $\gamma_{0}=\gamma_{1}=0.5$. The VA and the full numerical calculations have the value of $\mu=1$ fixed to the same value for $\tau$ $=0$, implying a small shift of $A(0)$, as shown by the results.

defocusing cycles connect the action of the periodically varying in the space with the inelastic three-body interactions. In a linear conservative optical lattice, with inelastic three-body interactions, the focusing-defocusing oscillations have been studied in Ref. [43]. Such oscillations have been studied in Ref. [44], using a harmonic trap with inelastic three-body interactions.

After the collapse is arrested, as observed in Fig. 6, the spreading out of the pulse can be compensated by an adiabatic variation of the background scattering length, considering the time variation of $\gamma_{0}$. When we consider a time dependent $\gamma_{0}$, as given in Eq. (11), the feeding term parameter $\alpha_{f}$ should be zero, because one can show (with a redefinition of the wave function) that it has a similar effect. In Fig. 8, we show our full numerical results confirming the stabilization of the condensate after the collapse was arrested. The mechanism of this stabilization was given by an appropriate tuning of the parameters $\alpha$ and $\tau_{c}$ of Eq. (11).

About the dissipative dynamics presented in this section, we should also add that effects of the lattice can also be observed in uniform systems. The variational approach can

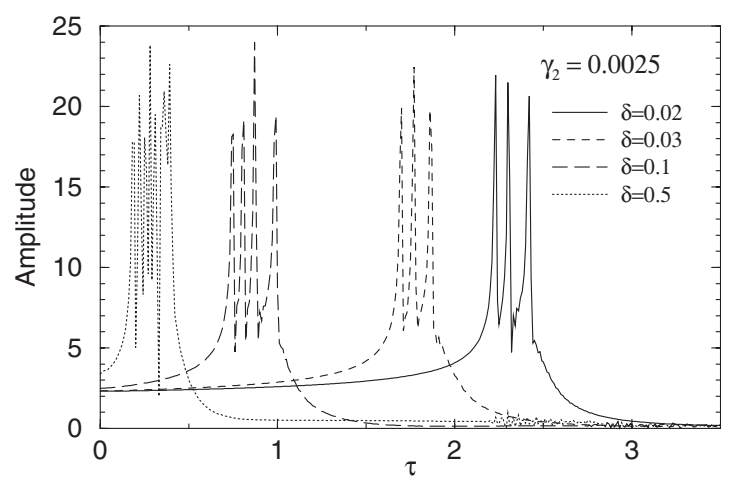

FIG. 7. Results for the amplitude as a function of $\tau$, showing the collapsing behavior for $\kappa=4 \pi$. As in Fig. $6, \gamma_{0}=\gamma_{1}=0.5$. The dissipation is fixed to $\gamma_{2}=0.0025$ and $\delta$ is increasing from 0.02 to 0.5 . 


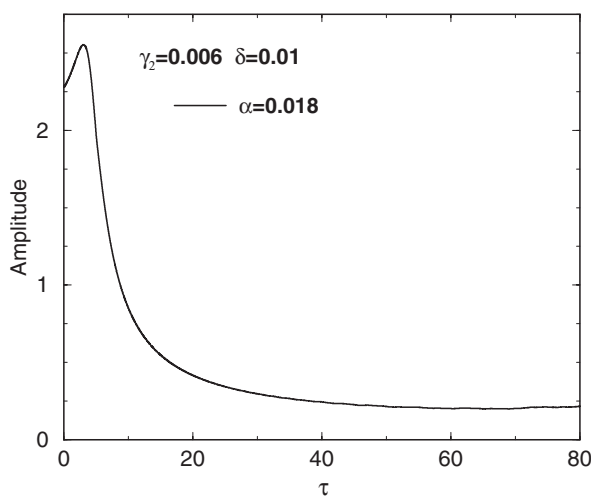

FIG. 8. Soliton stabilization via compression effect, with $\tau_{c}=5$ and $\alpha=0.018$ in Eq. (11). As in Figs. 6 and 7, $\gamma_{0}=\gamma_{1}=0.5$. The other parameters are $\gamma_{2}=0.006, \delta=0.01$, and $\kappa=4 \pi$.

describe dissipative dynamics between narrow and broad solitons relative to the lattice spacing $2 \pi / \kappa$. In the limit of a narrow soliton, we have a cubic dissipative term. In the opposite limit of a broad soliton, the averaged GPE contains an effective quintic imaginary term, providing a different dissipative dynamics. This effect appears with the replacement of $i \gamma_{2} \cos (\kappa x)|u|^{2} u$ by $i \Gamma_{3}|\bar{u}|^{4} \bar{u}$, with $\Gamma_{3}=3 \gamma_{2}^{2} /\left(2 \kappa^{2}\right)$ and $\bar{u}$ a field averaged over rapid modulations. Thus renormalization of the parameter corresponding to effective three-body losses, $\Gamma_{3}$, can be controlled by the values of the period $2 \pi / \kappa$ and amplitude $\gamma_{2}$ of the nonlinear modulations. The number of cycles reduces as $\Gamma_{3}$ grows and for large values of the damping, with the soliton decaying monotonically. These observations are also confirmed by the existence of focusingdefocusing cycles [44] in 3D BEC in a harmonic trap with two- and three-body inelastic processes.

\section{CONCLUSION}

Dynamics and stability of matter-wave solitons in the mixture of conservative and dissipative nonlinear optical lat- tices are investigated, considering 2D BEC, with 1D conservative plus dissipative nonlinear optical lattices.

In the first part of this work, we analyzed conservative systems, with nonlinear optical lattices, for attractive and repulsive condensates. The role of the scales when calculating the observables as the chemical potential and the widths was clarified. Our conclusion is that, in a 2D system, a nonlinear periodic lattice in one direction by itself cannot give stable solutions, satisfying the VK criterion [36]. Such a periodic lattice in the $x$ direction cannot compensate for the collapsing effect which results from the other dimension. We verify that stable solutions can be obtained by controlling the soliton with a harmonic trap in the $y$ direction. For repulsive condensates, the $2 \mathrm{D}$ stable soliton can exist in the geometry with 1D nonlinear optical lattices in one direction and harmonic trap in the other direction.

In the second part of the work we analyze the dynamics of the above 2D system, with periodic nonlinearity in the $x$ direction and without trap in the $y$ direction, when we add nonconservative nonlinear optical lattice terms. We show that the collapse of the condensate can be arrested by a dissipative periodic nonlinearity. To study the evolution of the 2D wave packet we apply the time-dependent variational approach. To compensate the wave packet broadening, the adiabatic time variation of scattering length is used. It is shown that the metastable dissipative soliton can exist in a 2D condensate with 1D periodic nonlinearity. Analytical predictions are confirmed by the numerical simulations of a full 2D GP equation.

\section{ACKNOWLEDGMENTS}

We thank Fundação de Amparo à Pesquisa do Estado de São Paulo for partial support. L.T. and A.G. also thank Conselho Nacional de Desenvolvimento Científico e Tecnológico for partial support.
[1] F. Kh. Abdullaev, A. Gammal, A. M. Kamchatnov, and L. Tomio, Int. J. Mod. Phys. B 19, 3415 (2005).

[2] B. A. Malomed, Soliton Management in Periodic Systems (Springer, New York, 2006).

[3] I. Gabitov and S. K. Turitsyn, Opt. Lett. 21, 327 (1996).

[4] N. J. Smith et al., Electron. Lett. 32, 54 (1996).

[5] F. Kh. Abdullaev, B. B. Baizakov, and M. Salerno, Phys. Rev. E 68, 066605 (2003).

[6] L. Bergé et al., Opt. Lett. 25, 1037 (2000).

[7] M. Owner-Petersen and A. Goncharov, J. Opt. Soc. Am. B 19, 537 (2002).

[8] Z. Y. Xu, Y. V. Kartashov, and L. Torner, Phys. Rev. Lett. 95, 113901 (2005).

[9] F. Kh. Abdullaev, A. M. Kamchatnov, V. V. Konotop, and V. A. Brazhnyi, Phys. Rev. Lett. 90, 230402 (2003).

[10] F. Kh. Abdullaev, J. G. Caputo, R. A. Kraenkel, and B. A. Malomed, Phys. Rev. A 67, 013605 (2003).

[11] H. Saito and M. Ueda, Phys. Rev. Lett. 90, 040403 (2003); H.
Saito and M. Ueda, Phys. Rev. A 70, 053610 (2004).

[12] G. D. Montesinos, V. M. Perez-Garcia, and P. J. Torres, Physica D 191, 193 (2004).

[13] V. Zharnitsky and D. Pelinovsky, Chaos 15, 037105 (2005).

[14] F. Kh. Abdullaev, E. N. Tsoy, B. A. Malomed, and R. A. Kraenkel, Phys. Rev. A 68, 053606 (2003).

[15] F.Kh. Abdullaev and J. Garnier, Phys. Rev. E 72, 035603(R) (2005).

[16] P. G. Kevrekidis, D. E. Pelinovsky, and A. Stefanov, J. Phys. A 39, 479 (2006); M. A. Porter, P. G. Kevrekidis, B. A. Malomed, and D. J. Frantzeskakis, Physica D 229, 104 (2007).

[17] S. K. Adhikari, Phys. Rev. A 69, 063613 (2004).

[18] Q. Quraishi, S. T. Cundiff, B. Ilan, and M. J. Ablowitz, Phys. Rev. Lett. 94, 243904 (2005).

[19] M. Centurion, M. A. Porter, P. G. Kevrekidis, and D. Psaltis, Phys. Rev. Lett. 97, 033903 (2006).

[20] P. J. Torres, Nonlinearity 19, 2103 (2006).

[21] A. Ciattoni, C. Rizza, E. DelRe, and E. Palange, Phys. Rev. 
Lett. 98, 043901 (2007).

[22] F. Kh. Abdullaev and M. Salerno, J. Phys. B 36, 2851 (2003).

[23] F. Kh. Abdullaev, A. Gammal, and L. Tomio, J. Phys. B 37, 635 (2004).

[24] G. Theocharis, P. Schmelcher, P. G. Kevrekidis, and D. J. Frantzeskakis, Phys. Rev. A 72, 033614 (2005).

[25] F.Kh. Abdullaev and J. Garnier, Phys. Rev. A 72, 061605(R) (2005).

[26] H. Sakaguchi and B. A. Malomed, Phys. Rev. E 72, 046610 (2005); 73, 026601 (2006).

[27] J. Garnier and F. Kh. Abdullaev, Phys. Rev. A 74, 013604 (2006).

[28] J. Belmonte-Beitia, V. M. Perez-Garcia, V. Vekslerchik, and P. J. Torres, Phys. Rev. Lett. 98, 064102 (2007).

[29] P. O. Fedichev, Yu. Kagan, G. V. Shlyapnikov, and J. T. M. Walraven, Phys. Rev. Lett. 77, 2913 (1996).

[30] M. Theis, G. Thalhammer, K. Winkler, M. Hellwig, G. Ruff, R. Grimm, and J. H. Denschlag, Phys. Rev. Lett. 93, 123001 (2004).

[31] G. Fibich, Y. Sivan, and M. I. Weinstein, Physica D 217, 31 (2006); Y. Sivan, G. Fibich, and M. I. Weinstein, Phys. Rev. Lett. 97, 193902 (2006).
[32] Y. V. Bludov and V. V. Konotop, Phys. Rev. A 74, 043616 (2006).

[33] F. Kh. Abdullaev, A. A. Abdumalikov, and R. M. Galimzyanov, Phys. Lett. A 367, 149 (2007).

[34] G. Dong and B. Hu, Phys. Rev. A 75, 013625 (2007).

[35] G. Fibich, SIAM J. Appl. Math. 61, 1680 (2001).

[36] N. G. Vakhitov and A. A. Kolokolov, Radiophys. Quantum Electron. 16, 783 (1973).

[37] L. Bergé, Phys. Rep. 303, 259 (1998).

[38] F. Kh. Abdullaev, A. Gammal, L. Tomio, and T. Frederico, Phys. Rev. A 63, 043604 (2001).

[39] P. D. Drummond and K. V. Kheruntsyan, Phys. Rev. A 63, 013605 (2000).

[40] M. Brtka, A. Gammal, and L. Tomio, Phys. Lett. A 359, 339 (2006).

[41] N. N. Akhmediev and A. Ankiewicz, Solitons: Nonlinear Pulses and Beams (Chapman and Hall, London, 1997).

[42] V. S. Filho, F. Kh. Abdullaev, A. Gammal, and L. Tomio, Phys. Rev. A 63, 053603 (2001).

[43] F. Kh. Abdullaev and M. Salerno, Phys. Rev. A 72, 033617 (2005).

[44] H. Saito and M. Ueda, Phys. Rev. Lett. 86, 1406 (2001). 